\title{
Meta-analysis of the clinical efficacy of FOLFIRINOX in the treatment of inoperable advanced pancreatic cancer and gemcitabine-based regimen
}

\author{
Daoming Zhang ${ }^{1}$, Yongfa Zheng ${ }^{1}$, Xufeng Guo ${ }^{1}$, Tian Tang ${ }^{1^{\star}}$, Hui Ren ${ }^{2^{\star}}$ \\ ${ }^{1}$ Department of Oncology, Renmin Hospital of wuhan University, wuhan University, wuhan 430060, Hubei, China; \\ ${ }^{2}$ Department of General Surgery, The China-Japan Union Hospital of Jilin University, Changchun 130000, Jilin, China \\ *Corresponding author: Professor Tian Tang, Department of Oncology, Renmin Hospital of wuhan University, \\ wuhan University, wuhan 430060, Hubei, China; E-mail: 793647053@ qq.com; Professor Hui Ren, Department of \\ General Surgery, The China-Japan Union Hospital of Jilin University, 2 Xiantai Street, Changchun, Jilin 130000, \\ China; E-mail: hren@jlu.edu.cn
}

Received May 28, 2019; Accepted November 17, 2019

\begin{abstract}
Aim: To compare the efficacy and adverse reactions of FOLFIRINOX and gemcitabine-containing regimen in chemotherapy for inoperable advanced pancreatic cancer.

Methods: The clinical studies that met the inclusion criteria were searched in domestic and foreign databases such as EMbase, Cochrane library, Pubmed, MEDLINE, CNKI, and Wanfang Data, and the quality of the included studies was evaluated according to the Cochrane System Evaluation Manual 5.0. We performed meta-processing analysis on related indicators through Revman 5.1 software.

Results: A total of 7 kinds of clinical research literature were included, with a total of 2508 patients. The objective response rate, 1-year overall survival rate, and 6-month disease-free survival rate of the FOLFIRINOX regimen were better than those of the gemcitabine-containing regimen. The RR values were 1.6 (95\% CI 1.03-2.47), 1.56 (95\% CI 1.24-1.96), and 1.71 (95\% CI 1.21-2.41). In terms of grade 3-4 adverse events, the incidence of vomiting $(R R=1.4895 \% C I 1.18-1.87)$ and diarrhea $(R R=3.0295 \%$ CI 1.23-7.44) in the FOLFIRINOX regimen was higher than in the gemcitabine-containing regimen. There was no statistical difference between the two groups in terms of neutropenia, thrombocytopenia, and fever.

Conclusions: In the chemotherapy of inoperable advanced pancreatic cancer, the FOLFIRINOX regimen is superior to a gemcitabine-containing regimen in terms of efficiency and survival, and adverse reactions have increased.
\end{abstract}

Key Words: pancreatic cancer; chemotherapy; FOLFIRINOX; gemcitabine; Meta-analysis

\section{INTRODUCTION}

Pancreatic cancer is a common malignant tumor of the digestive system in China, and the incidence rate is increasing year by year. According to data released by the Cancer Center of China in 2017, the incidence rate ranked 10th and the tumor-related mortality rate ranked 6th. ${ }^{1}$ Due to the insidious onset of the disease, there are no obvious specific symptoms, and most patients have lost the opportunity for radical surgery or have distant metastasis at the time of diagnosis. As the "king of cancer", the 5-year survival rate is less than $5 \%{ }^{2}$ Finding a systemic treatment with a positive effect is of great significance for the treatment of advanced pancreatic cancer. Gemcitabine is currently recognized as a potent chemotherapy drug for pancreatic cancer. Many clinical trials have explored the efficacy of a combination regimen based on gemcitabine, but the

This is an open access journal, and articles are distributed under the terms of the Creative Commons Attribution-NonCommercial-ShareAlike 4.0 License, which allows others to remix, tweak, and build upon the work non-commercially, as long as appropriate credit is given and the new creations are licensed under the identical terms.

For reprints contact: weda-h@weda-h.org

How to cite this article: Zhang DM, Zheng YF, Guo XF, Tang T, Ren H. Meta-analysis of the clinical efficacy of FOLFIRINOX in the treatment of inoperable advanced pancreatic cancer and gemcitabine-based regimen. J ADV HEALTH 2019; 1(4): 281-286. 
benefits compared with gemcitabine alone are not satisfactory. Among them, the most effective combination of albumin-bound paclitaxel (NAB) increased the OS by only 1.8 months. ${ }^{3}$ The results of the PRODIGE 4/ACCORD 11 research confirmed that folinic acid, fluorouracil, irinotecan and oxaliplatin (FOLFIRINOX) regimen is approximately 4.3 months longer than gemcitabine in terms of median survival, ${ }^{4}$ but the combination of the four drugs(folinic acid, fluorouracil, irinotecan and oxaliplatin) may have an increased toxic side effect, limiting the application of the FOLFIRINOX regimen. Our study, through meta-analysis, evaluated the efficacy and toxic side effects of FOLFIRINOX and gemcitabine-containing regimens in advanced pancreatic cancer with lost surgical opportunities intended to provide a reference for clinical work.

\section{MATERIALS AND METHODS}

\section{Literature search}

Search for the related keywords "Pancreatic Neoplasm; Pancreas Neoplasm; Pancreas Cancer; FOLFIRINOX; Gemcitabine" in domestic and foreign databases such as EMbase, Cochrane library, Pubmed, MEDLINE, CNKI, and Wanfang Data. The time interval was set from January 2010 to February 2019. We manually searched for the literature to obtain journal articles and, if necessary, contact the relevant authors and companies.

\section{Literature screening}

The two researchers independently read the searched literature titles and abstracts, Firstly we screened out the documents that did not meet the inclusion criteria, and further screened articles that were more relevant to the research, and manually searched for the original documents. Combined with the exclusion criteria, the clinical study literature included in the meta-analysis was determined.

\section{Inclusion criteria and exclusion criteria}

We collected randomized, controlled clinical studies and retrospective studies published from January 2010 to February 2019 using the FOLFIRINOX regimen for the treatment of inoperable advanced pancreatic cancer versus the clinical efficacy of the gemcitabine regimen. Inclusion criteria: (1) Research subjects were patients with locally advanced pancreatic cancer who lost the opportunity of radical surgery or patients with metastatic pancreatic cancer; (2) The purpose was to compare the clinical efficacy and side effects of FOLFIRINOX chemotherapy regimen with gemcitabine-containing chemotherapy regimen; (3) All research data include complete research data, specific patient cases and efficacy evaluation; (4) If there are multiple sets of clinical data, select the two sets of data related to this meta-analysis. Exclusion criteria: (1) literature review and meta-analysis; (2) analysis of the same clinical trial data and repeated publications; (3) trial methods including literature on combined radiotherapy, minimally invasive, and conversion therapy; (4) The literatures with too few cases, less than 25 cases; (5) literatures that does not provide sufficient data.

\section{Quality evaluation}

All included literatures were evaluated for quality according to the article Quality Evaluation Standard (NOS) scale.

\section{Statistical processing}

After data extraction and sorting, the Metaman5.1 software provided by the Cochrane Collaboration Network was used to perform meta-processing analysis on related indicators. Count data were statistically described using relative risk (RR) and 95\% confidence interval $(95 \% \mathrm{CI})$. The heterogeneity of $P$ value and $I^{2}$ is judged by Q test: If the result was $P \leqslant 0.1, I^{2}>50 \%$, indicating that the heterogeneity is large, using a random effect model; if the data suggested $P>0.1, I^{2} \leqslant 50 \%$, indicating that the heterogeneity is small, using a fixed-effect model. $P<0.05$ indicates statistical significance.

\section{OUTCOME}

\section{Literature search results}

243 related articles were obtained through keyword search. After layer-by-layer screening based on the inclusion criteria and exclusion criteria, 5 English-compliant documents and 2 Chineselanguage documents (Figure 1) was finalized.

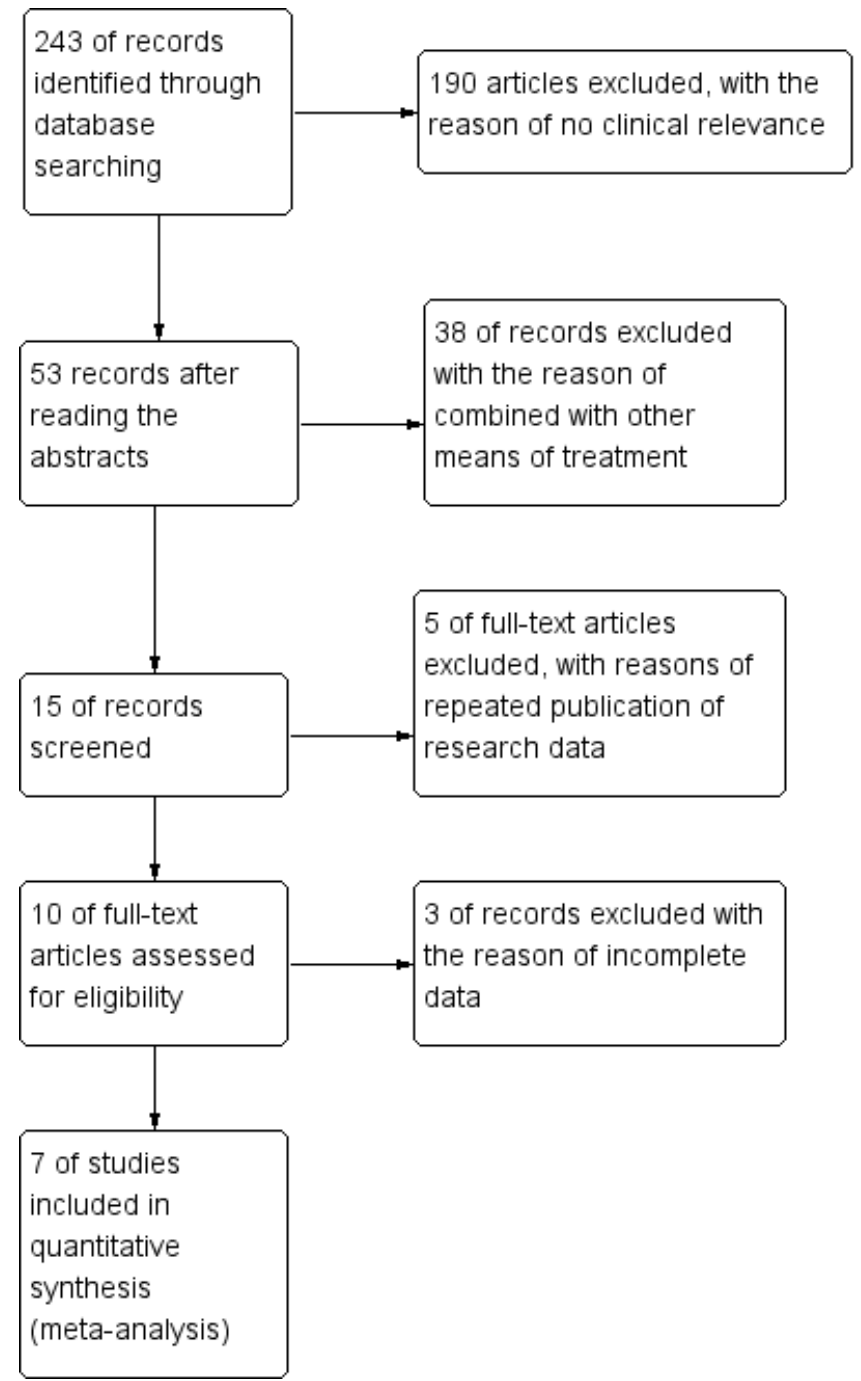

Figure 1 Flow chart showing the selection of the studies 


\section{Inclusion of basic characteristics and quality evaluation of the literature}

Among the 7 articles included, 4 were prospective, randomized controlled trials, and 3 were retrospective analyses of clinical data. $^{5-11}$ Three kinds of literature presented FOLFIRINOX and different intergroup studies containing the gemcitabine protocol, which were grouped to collect data and analysis. The gemcitabine- containing regimens involved include the gemcitabine monotherapy regimen, the gemcitabine plus capecitabine regimen, the gemcitabine plus tegafur regimen, and the gemcitabine combined with the albumin-bound paclitaxel regimen. A total of 3346 patients, including 1090 patients receiving FOLFIRINOX chemotherapy regimen, 2256 patients receiving gemcitabine chemotherapy regimen (Table 1). The NOS scores included in the literature were all $\geqslant 6$ points, and the quality was appropriately acceptable.

Table1 Basic characteristics and quality evaluation of the included studies

\begin{tabular}{|c|c|c|c|c|c|}
\hline Author & Year of publication & Country & Period of inclusion & Number of Patients & NOS score \\
\hline Thierry Conroy ${ }^{4}$ & 2011 & France & Dec 2005 -Oct 2009, & 342 & 8 \\
\hline Zemian Chen ${ }^{5}$ & 2018 & China & May-2016 May 2018 & 68 & 6 \\
\hline Yuan Qian ${ }^{6}$ & 2018 & China & Jan 2014 - Dec 2017 & 70 & 7 \\
\hline Sunnie $\mathrm{Kim}^{7}$ & 2018 & USA & Apr 2015 -Dec 2015 & 654 & 7 \\
\hline Takeshi Terashima ${ }^{8}$ & 2018 & Japan & Jan 2009 -Jul 2015 & 432 & 6 \\
\hline Susanna Hegewisch-Becker ${ }^{9}$ & 2019 & Germany & Feb 2014- Jun 2017 & 1045 & 6 \\
\hline Muhammad Ahsan Javed ${ }^{10}$ & 2019 & Europe & Jan 2012 -Dec 2015 & 735 & 6 \\
\hline
\end{tabular}

\section{Meta-analysis results}

\section{Objective response rate}

The objective response rate was mentioned in 5 kinds of literature (Figure 2). The meta-analysis showed that the objective response rate of the FOLFIRINOX regimen (Experimental) was higher than that of the gemcitabine-containing regimen (Control). The
$R R$ was 1.6 (95\% CI 1.03-2.47), $P=0.04$, which was statistically significant.

\section{Median survival}

The median overall survival time was reported in all kinds of literature, and 4 reported median progression-free survival time (Table 2).

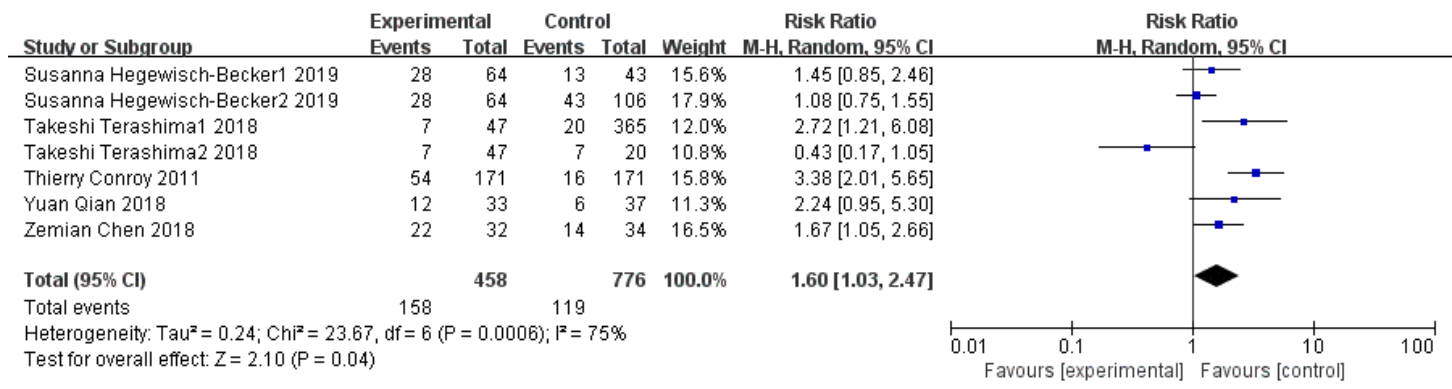

Figure 2 Forest plot displaying the results of Meta-analysis of Objective response rates

Table 2 Median overall and progression-free survival

\begin{tabular}{|c|c|c|c|c|}
\hline \multirow{2}{*}{ Study } & \multicolumn{2}{|c|}{ Median overall survival (months; 95\% CI) } & \multicolumn{2}{|c|}{ Median progression-free survival (months; $95 \%$ CI) } \\
\hline & Experimental & Control & Experimental & Control \\
\hline Thierry Conroy & $11.1(9.0-13.1$ & $6.8(5.5-7.6)$ & $6.4(5.5-7.2)$ & $3.3(2.2-3.6)$ \\
\hline Zemian Chen & 10 & 8 & 9 & 7 \\
\hline Yuan Qian & $13.5(6.4-20.7)$ & $8.3(6.4-10.2)$ & $6.1(1.2-11)$ & $4.3(3.1-5.5)$ \\
\hline Sunnie Kim & 13.8 & 12.6 & Not reached & Not reached \\
\hline Takeshi Terashimal & 10.3 & 7.5 & Not reached & Not reached \\
\hline Takeshi Terashima2 & 10.3 & 9.9 & Not reached & Not reached \\
\hline Susanna Hegewisch-Becker1 & $11.3(10.5-12.5)$ & $6.8(6.1-9.0)$ & $6.3(5.5-6.9)$ & $4.6(3.7-5.2)$ \\
\hline Susanna Hegewisch-Becker2 & $11.3(10.5-12.5)$ & $9.1(8.2-10.1)$ & $6.3(5.5-6.9)$ & $5.6(5.0-6.2)$ \\
\hline Muhammad Ahsan Javed1 & $9.9(8.4-12.6)$ & $4.9(4.4-5.6)$ & Not reached & Not reached \\
\hline Muhammad Ahsan Javed 2 & $9.9(8.4-12.6)$ & $7.9(6.2-10.0)$ & Not reached & Not reached \\
\hline
\end{tabular}


1-year overall survival rate

Six articles reported a 1-year overall survival rate (Figure 3 ). The meta-analysis showed that 1-year overall survival rate of the FOLFIRINOX regimen group was higher than that of the gemcitabine-containing regimen. The $R R$ was $1.56(95 \% C I$ 1.24-1.96), $P<0.001$, which was statistically significant.

\section{6-month disease-free survival rate}

4 reports reported 6-month disease-free survival (Figure 4). The meta-analysis showed that the FOLFIRINOX regimen had a 6-month disease-free survival rate higher than that of gemcitabine. Program group. The $R R$ was 1.71 (95\% CI 1.21-2.41), $P=0.002$, which was statistically significant.

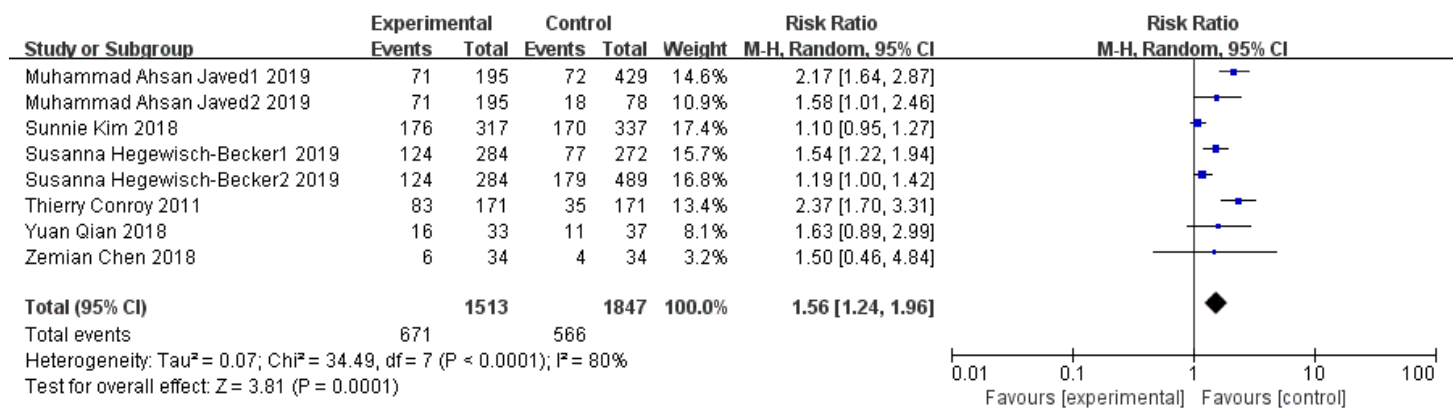

Figure 3 Forest plot displaying the results of Meta-analysis of 1-year overall survival rate

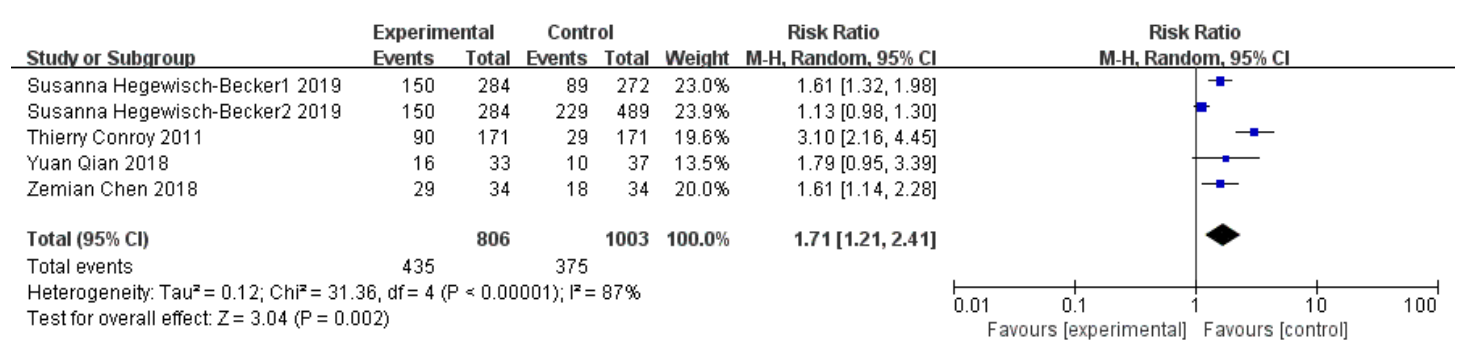

Figure 4 Forest plot displaying the results of Meta-analysis of 6 months progress free survival rate

\section{3/4 toxic side effects}

Toxic side effects included in the analysis were: neutropenia, thrombocytopenia, vomiting, diarrhea, infection and fever. Five kinds of literature reported neutropenia, thrombocytopenia, and vomiting. Four reports reported the incidence of diarrhea and fever. The meta-analysis showed no significant differences in the incidence of neutropenia (Figure 5), thrombocytopenia (Figure 6), and infection with fever (Figure 7) in the FOLFIRINOX regimen and the gemcitabine-containing regimen. The incidence of vomiting (Figure 8) $(R R=1.4895 \% C I$ 1.18-1.87) and diarrhea (Figure 9$)(R R=3.0295 \%$ $C I$ 1.23-7.44) was higher in the FOLFIRINOX regimen.

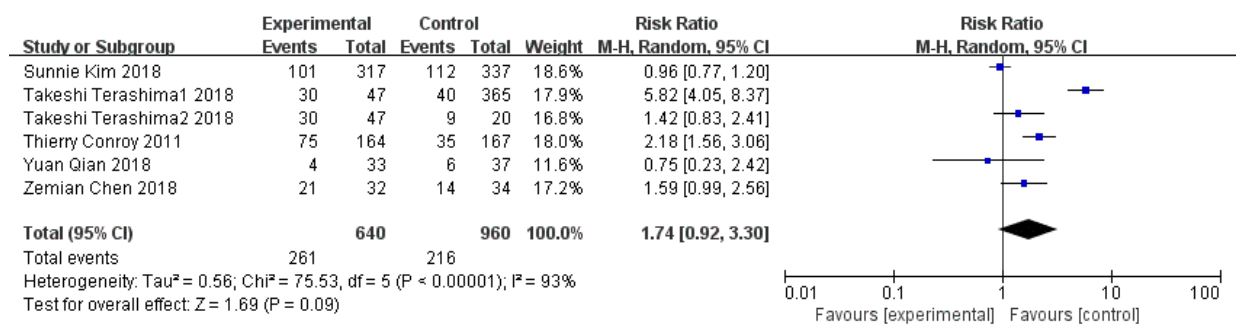

Figure 5 Forest plot displaying the results of Meta-analysis of grade 3-4 neutropenia

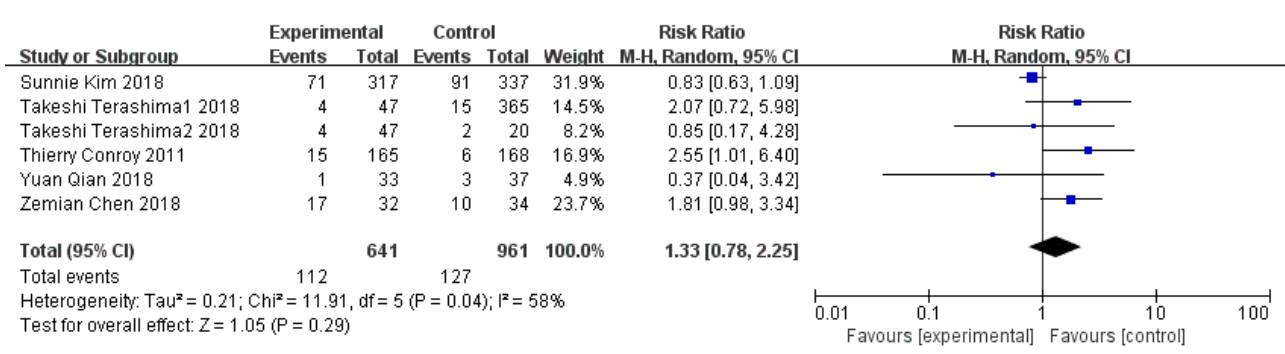

Figure 6 Forest plot displaying the results of Meta-analysis of grade 3-4 Thrombocytopenia 


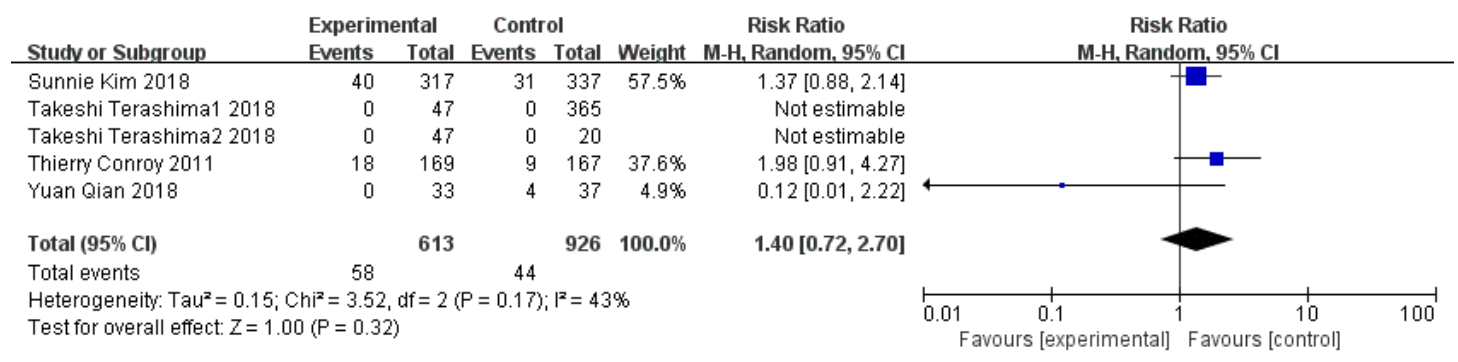

Figure 7 Forest plot displaying the results of Meta-analysis of grade 3-4 infectious

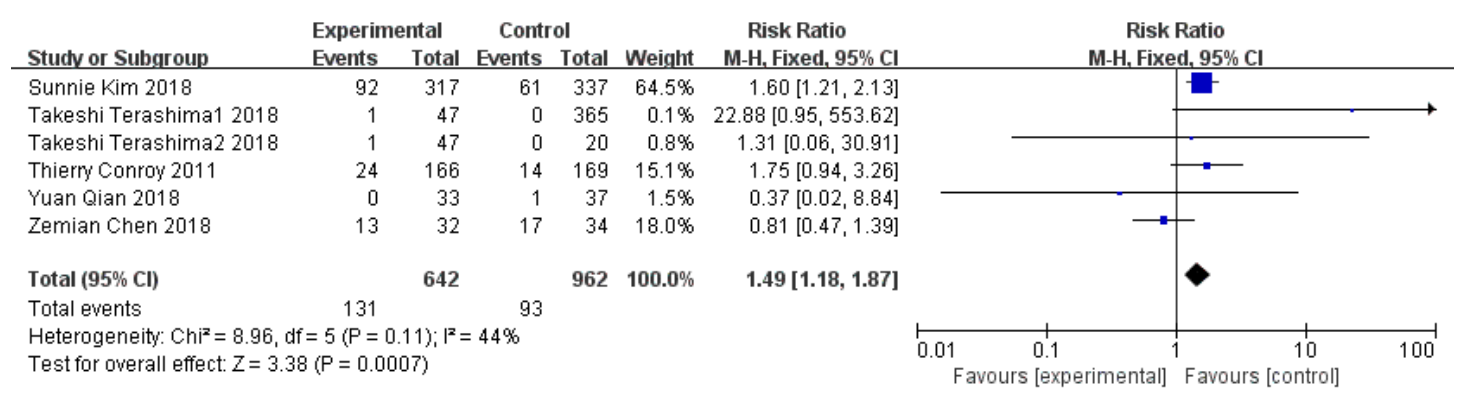

Figure 8 Forest plot displaying the results of Meta-analysis of grade 3-4 vomiting

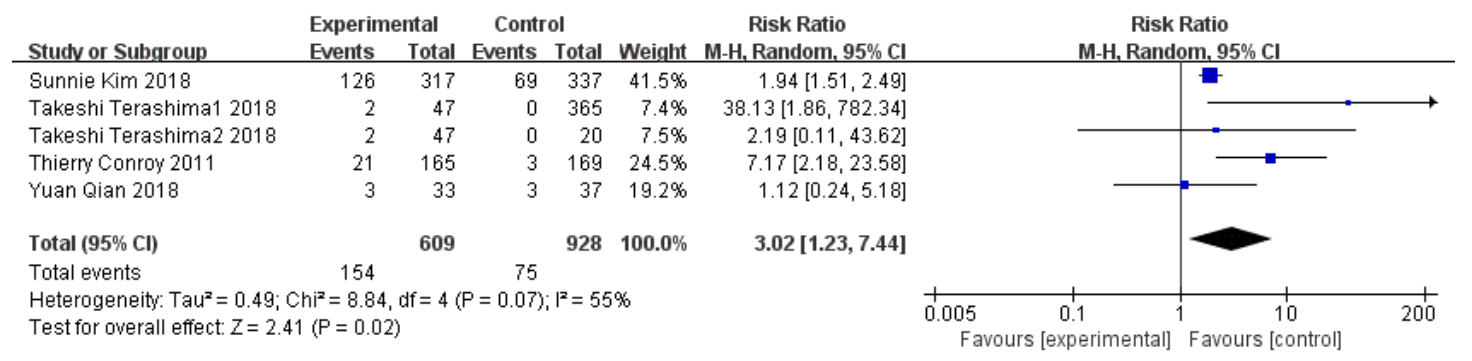

Figure 9 Forest plot displaying the results of Meta-analysis of grade 3-4 Diarrhea

\section{DISCUSSION}

In the systemic treatment of advanced pancreatic cancer, the gemcitabine combination regimen includes $S-1$, capecitabine, paclitaxel, albumin paclitaxel, erlotinib. The recommended level of gemcitabine combined with albumin paclitaxel regimen and the FOLFIRINOX regimen in the NCCN guidelines is higher, ${ }^{11}$ and it is noted that the gemcitabine combined with the paclitaxel regimen is recommended for patients with ECOG $\leqslant 2$ in the first-line regimen, and the FOLFIRINOX regimen is recommended for patients with ECOG $\leqslant 1$. Such stratification recommendations mainly consider the higher incidence of toxic side effects in the FOLFIRINOX regimen, and the physical strength of the patients included in the PRODIGE 4/ACCORD 11 study was also great, which ensures the successful completion of chemotherapy in the study. However, in the real-world retrospective analysis of Kim S, there was no statistically significant difference in the survival benefit of FOLFIRINOX or gemcitabine combined with albumin paclitaxel. ${ }^{7}$ This may be related to older patients, higher physical scores, and more complications in the gemcitabine-conjugated albumin paclitaxel chemotherapy group. Javed MA conducted a multivariate analysis of the data to conclude that males and poor physical strength were factors associated with poor prognosis. ${ }^{10}$

In terms of safety, this meta-analysis included five indicators of neutropenia, thrombocytopenia, vomiting, diarrhea, and infection fever. The results suggest that there is no significant difference in hematological toxicity between the two groups. However, the incidence of gastrointestinal side effects in the FOLFIRINOX group was higher, which may be closely related to the application of irinotecan. Irinotecan is a semi-synthetic soluble camptothecin derivative that inhibits DNA replication and transcription by inhibiting DNA topoisomerase I, leading to tumor cell death. It also adversely affects other tissues with active cell proliferation, such as intestinal mucosa and bone marrow. ${ }^{12}$ Irinotecanassociated diarrhea includes early-onset diarrhea and delayed diarrhea. Early-onset diarrhea is one of the most common manifestations of irinotecan, specifically inhibiting cholinesteraseinduced cholinergic syndrome, and delayed diarrhea is caused by its unique dose-limiting toxicity. ${ }^{13}$ In order to reduce the incidence of grade 3/4 toxicity in the FOLFIRINOX regimen, many scholars have improved the regimen by reducing the dose of irinotecan and eliminating the intravenous injection of $400 \mathrm{mg} / \mathrm{m}^{2}$ of fluorouracil. Studies have confirmed that the improved program has achieved comparable efficacy to the original regimen while reducing toxic and side effects, which is worth exploring and promoting. ${ }^{14-15}$ In the literature included in this meta-analysis, the authors of the two Chinese kinds of literature used the modified FOLFIRINOX regimen to improve the incidence of myelosuppression and gastrointestinal reactions. In addition, only two articles reporting the incidence of neurotoxicity were not included in the meta- 
analysis. However, both reports suggest that the incidence of neurotoxicity in the FOLFIRINOX regimen is higher, which may have a greater relationship with the application of oxaliplatin. Due to the differences between Asian humans and Westerners, largescale Asian population research trials are meaningful for exploring the clinical benefits of specific populations. The JCOG1407 study is a prospective controlled trial of the comparatively improved FOLFIRINOX regimen and gemcitabine combined with albumin paclitaxel in 36 Japanese medical institutions. ${ }^{16}$ The results are worth looking forward to.

There are many limitations in this meta-analysis: (1) Language restrictions are Chinese and English, and related literature in other languages has not been included, resulting in missed research. (2) Due to the insufficient number of prospective controlled trials in the relevant studies, retrospective analysis literature was included in this study. Retrospective studies often lacked a good baseline of data and had a large number of confounding factors. (3) In the study, some of the literature samples are small, and there is no mention of the index quality evaluation indicators such as allocation concealment and blindness. (4) A number of research indicators are heterogeneous when performing the meta-analysis. (5) after the failure of first-line treatment, the second-line treatment program is mixed, which has an uncontrollable effect on survival follow-up.

\section{acknowledement}

Not applicable.

\section{REFERENCES}

1. Chen W, Zheng R, Zhang S. Cancer incidence and mortality in China in 2013: an analysis based on urbanization level. Chin J Cancer Res 2017; 29(1): 1-10

2. Eser S, Schnieke A, Schneider G, Saur D. Oncogenic KRAS signaling in pancreatic cancer. Br J Cancer 2014; 111(5): 817-822.

3. Von Hoff DD, Ervin T, Arena FP, Chiorean EG, Infante J, Moore M, Seay T, Tjulandin SA, Ma WW, Saleh MN, Harris M, Reni M, Dowden S, Laheru D, Bahary N, Ramanathan RK, Tabernero J, Hidalgo M, Goldstein D, Van Cutsem E, Wei X, Iglesias J, Renschler MF. Increased survival in pancreatic cancer with nab-paclitaxel plus gemcitabine. N Engl J Med 2013; 369(18): 1691-1703.

4. Conroy T, Desseigne F, Ychou M, Bouché O, Guimbaud R, Bécouarn Y, Adenis A, Raoul JL, Gourgou-Bourgade S, de la Fouchardière C, Bennouna J, Bachet JB, Khemissa-Akouz F, Péré-Vergé D, Delbaldo C, Assenat E, Chauffert B, Michel P, Montoto-Grillot C, Ducreux M; Groupe Tumeurs Digestives of Unicancer; PRODIGE Intergroup. FOLFIRINOX versus gemcitabine for metastatic pancreatic cancer. $N$ Engl J Med 2011; 364(19): 1817-1825.

5. Chen ZM, Su GZ, Zhao CY. Comparative Study of Gemcitabine and Capecitabine in the Treatment of Stage IV Pancreatic Cancer with FOLFIRINOX Protocol. Medical Innovation of China 2018; 15(30):37-41.

6. Qian Y, Zheng S, Jia CK. Comparison of modified FOLFIRINOX chemotherapy and gemcitabine-based chemotherapy in the treatment of advanced pancreatic cancer. Journal of Practical Oncology 2018; 33(3): 237-245.
7. Kim S, Signorovitch JE, Yang H, Patterson-Lomba O, Xiang CQ, Ung B , Parisi M, Marshall JL. Comparative Effectiveness of nab-Paclitaxel Plus Gemcitabine vs FOLFIRINOX in Metastatic Pancreatic Cancer: A Retrospective Nationwide Chart Review in the United States. Adv Ther 2018; 35(10): 1564-1577.

8. Terashima T, Yamashita T, Sakai A, Ohta H, Hinoue Y, Toya D, Kawai H, Yonejima M, Urabe T, Noda Y, Mizukoshi E, Kaneko S. Treatment patterns and outcomes of unresectable pancreatic cancer patients in real-life practice: a region-wide analysis. Jpn J Clin Oncol 2018; 48(11): 966-973.

9. Hegewisch-Becker S, Aldaoud A, Wolf T, Krammer-Steiner B, Linde $H$, Scheiner-Sparna $R$, Hamm $D$, Jänicke $M$, Marschner $N$, TPK-Group(Tumour Registry Pancreatic Cancer). Results from the prospective German TPK clinical cohort study: Treatment algorithms and survival of 1,174 patients with locally advanced, inoperable, or metastatic pancreatic ductal adenocarcinoma. Int J Cancer 2019; 144(5):981-90.

10. Javed MA, Beyer G, Le N, Vinci A, Wong H, Palmer D, Morgan RD, Lamarca A, Hubner RA, Valle JW, Alam S, Chowdhury S, Ma YT, Archibugi L, Capurso G, Maisonneuve P, Neesse A, Sund M, Schober M, Krug S. Impact of intensified chemotherapy in metastatic pancreatic ductal adenocarcinoma (PDAC) in clinical routine in Europe. Pancreatology 2019;19(1):97-104.

11. Tempero MA, Malafa MP, Al-Hawary M, Asbun H, Bain A, Behrman SW, Benson AB 3rd, Binder E, Cardin DB, Cha C, Chiorean EG, Chung V, Czito B, Dillhoff M, Dotan E, Ferrone CR, Hardacre J, Hawkins WG, Herman J, Ko AH, Komanduri S, Koong A, LoConte N, Lowy AM, Moravek C, Nakakura EK, O'Reilly EM, Obando J, Reddy S, Scaife C, Thayer S, Weekes CD, Wolff RA, Wolpin BM, Burns J, Darlow S. Pancreatic adenocarcinoma, version 2.2017, NCCN clinical practice guidelines in oncology. J Natl Compr Canc Netw 2017; 15(8): 1028-1061.

12. de Man FM, Goey AKL, van Schaik RHN, Mathijssen RHJ, Bins S. Individualization of irinotecan treatment:A review of pharmacokinetics, pharmacodynamics, and pharmacogenetics. Clin Pharmacokinet 2018; 57(10): 1229-1254

13. Yu QY, Wang HL, Wu T. A case of serious adverse effect induced by irinotecan. Chin Hosp Pharm J 2012; 32(9) :733-735.

14. Blazer M, Wu C, Goldberg RM, Phillips G, Schmidt C, Muscarella P, Wuthrick E, Williams TM, Reardon J, Ellison EC, Bloomston M, Bekaii-Saab T. Neoadjuvant modified(m) FOLFIRINOX for locally advanced unresectable (LAPC) and borderline resectable (BRPC) adenocarcinoma of thepancreas. Ann Surg Oncol 2015; 22(4): 1153-1159.

15. Stein SM, James ES, Deng Y, Cong X, Kortmansky JS, Li J, Staugaard C, Indukala D, Boustani AM, Patel V, Cha CH, Salem RR, Chang B, Hochster HS, Lacy J. Final analysis of a phase II study of modified FOLFIRINOX in locally advanced and metastatic pancreatic cancer. Br J Cancer 2016; 114(7): 737-743.

16. Mizusawa J, Fukutomi A, Katayama H, Ishii H, Ioka T, Okusaka T, Ueno H, Ueno M, Ikeda M, Mizuno N, Ozaka M, Fukuda H, Furuse J; Hepatobiliary and Pancreatic Oncology Group of the Japan Clinical Oncology Group. Protocol digest of randomized phase II study of modified FOLFIRINOX versus gemcitabine plus nab-paclitaxel combination therapy for locally advanced pancreatic cancer: Japan clinical oncology group study (JCOG1407). Pancreatology 2018; 18(7): 841-845. 